\title{
Genetic dissection of Drosophila myofibril formation: effects of actin and myosin heavy chain null alleles
}

\author{
Clifford J. Beall, ${ }^{1}$ Michael A. Sepanski, ${ }^{2}$ and Eric A. Fyrberg1 \\ ${ }^{1}$ Department of Biology, The Johns Hopkins University, Baltimore, Maryland 21218 USA; ${ }^{2}$ Department of Embryology, \\ Carnegie Institution of Washington, Baltimore, Maryland 21210 USA
}

\begin{abstract}
We used null mutations of Drosophila actin and myosin genes to investigate two aspects of myofibril assembly. First, we eliminated all actin or myosin in flight muscles to evaluate contributions of thick and thin filaments to sarcomere formation. Results demonstrate that thick and thin filament arrays can assemble independently but that both are essential for sarcomeric order and periodicity. Second, we examined how filament stoichiometry affects myofibril assembly. We find that heterozygotes for actin (Act88F) or myosin heavy chain (Mhc36B) null alleles have complex myofibrillar defects, whereas Mhc36B-/ + ; Act88F- + double heterozygotes have nearly normal myofibrils. These results imply that most defects observed in single heterozygotes are due to filament imbalances, not deficits, and suggest that thick and thin filament interactions regulate myofibrillar growth and alignment.
\end{abstract}

[Key Words: Drosophila; myofibril assembly; myosin; myofibrils; actin]

Received November 3, 1988; revised version accepted December 27, 1988.

Myofibril assembly is a complex process, details of which are poorly understood. During muscle development, thick and thin filaments form by polymerization of myosin, actin, and associated proteins. The two filament types become interdigitated in precise hexagonal arrays and anchored by transverse linkages in $M$ lines and $\mathrm{Z}$ discs. Some aspects of myofibril assembly and many ultrastructural features of myofibrils are known from immunocytochemical and electron microscopy studies (see, e.g., Shafiq 1963a,b; Fischman 1967, 1986; Auber 1969; Peng et al. 1981; Taylor et al. 1984; Sanger et al. 1986). However, neither these analyses nor biochemical and biophysical investigations of actin and myosin polymerization (see Pollard and Cooper 1986; Davis 1988) have yet answered many fundamental questions relating to mechanisms by which filaments are aligned and fastened, how their lengths are so uniformly regulated, and the role of myosin cross bridges in determining or stabilizing orientations of thick and thin filaments during myofibrillar assembly.

Genetic methods offer an effective means by which to dissect the program of myofibril assembly into its parts. In genetically tractable organisms such as Caenorhabditis elegans (for review, see Waterston and Francis 1985) and Drosophila melanogaster (for review, see Fyrberg 1989|, one can perturb or eliminate individual myofibrillar components using mutations and observe the resultant defects in vivo. If the mutant myofibrils differ from their wild-type counterparts in a significant and reproducible manner, then processes by which myofibrils normally form may be illuminated.
Drosophila indirect flight muscles (IFM) lend themselves well to genetic approaches because mutations that disrupt these fibers confer an easily recognized flightless phenotype without reducing fertility or viability. Among the mutants characterized previously are those affecting the sarcomeric myosin heavy chain gene $M h c 36 B$ and the flight muscle-specific actin gene Act $88 F$. Mhc $36 B$ transcripts are spliced via alternative pathways and thus specify several distinct myosin heavy chain isoforms, some of which accumulate only within flight muscles (Bernstein et al. 1986; B. George and C. Emerson, pers. comm.). Act $88 F$ is expressed only within flight muscles and encodes all actin of these fibers (Ball et al. 1987). Some of the myosin heavy chain and actin mutations are null, i.e., they specify no detectable protein. The Mhc36B mutation Ifm(2)2 is a flight musclespecific null allele that eliminates all myosin heavy chain mRNA and protein from these fibers (Mogami and Hotta 1981; Chun and Falkenthal 1988; C.J. Beall et al., unpubl.), whereas the Act88F mutation KM88 eliminates all actin mRNA and protein from these same muscles (Okamoto et al. 1986).

We have used myosin and actin null alleles to investigate two aspects of myofibril assembly. First, we eliminated actin filaments and ascertained whether myosin filaments formed ordered networks in their absence and, conversely, eliminated myosin and observed effects on formation of thin filament networks. Second, we examined whether alterations in myosin and actin stoichiometry affect the program of myofibril assembly. We and others have previously noted a surprising aspect of actin 
and myosin heavy chain null mutations: that they greatly reduce flight even in heterozygotes (Okamoto et al. 1986; O'Donnell and Bernstein 1988). One possible explanation is that the observed defects are due to underproduction of the encoded proteins. In this model, reduced levels of actin and myosin would lead to lowered numbers of the corresponding filaments and, hence, muscular weakness. However, it seemed equally possible that the observed defects are due to incorrect stoichiometry of nascent proteins leading to defective patterns of assembly. In this model, the muscle defects would be due to abnormal sarcomere structure and arrangement, rather than to reduced number of filaments. In this paper we present investigations of actin and myosin heavy chain gene null alleles that are designed to address both of the aforementioned issues. We have examined myosin heavy chain, actin, and myosin plus actin null mutants using electron microscopy. These results show that arrays of thick or thin filaments can assemble independently but that both are requisite for sarcomere order and periodicity. Our results further reveal that $\mathrm{M}$ lines form in the absence of thin filaments, whereas $\mathrm{Z}$ disc integrity is largely independent of thick filaments. We have also examined myofibril morphology in myosin, actin, and myosin plus actin null allele heterozygotes. Surprisingly, we find that double heterozygotes have more normal myofibrils than either single heterozygote. These results suggest that most defects

Figure 1. Flight muscle defects associated with actin and myosin heavy chain null allele homozygotes. (A) Electron micrographs of flight muscles from Act $88 F^{K M 88}$ homozygotes. Muscle fibers are devoid of thin filaments. In their absence, imperfectly aligned skeins of thick filaments form. $M$ lines can be discerned, demonstrating that many thick filaments are in lateral register. In cross sections (inset), thick filaments are sometimes perfectly packed into hexagonal arrays. Such precise packing is only seen when cores of thick filaments are solid. Because only central regions of thick filaments have solid cores, this result suggests that the $M$ line organizes or stabilizes the hexagonal packing. $(B)$ Flight muscles of $M h c 36 B^{I f m(2) 2}$ myosin heavy chain null allele homozygotes. No thick filaments are present, and in their absence pseudomyofibrils consisting of apparently continuous arrays of thin filaments anchored aperiodically within $\mathrm{Z}$ discs form. (C) Flight muscles of $M h c 36 B^{\text {Ifm(2)2 }}$; Act $88 F^{\text {KM88 }}$ double homozygotes. In the absence of both thin and thick filaments, nuclei and mitochondria are the only discernible organelles. T-tubule/sarcoplasmic reticulum dyads are either poorly developed or absent. Bar, $1 \mu \mathrm{m}$, except for the $A$ inset, where it equals $0.5 \mu \mathrm{m}$. For comparisons to wild-type myofibrils, refer to Figs. $3 \mathrm{~A}$ and $4 \mathrm{~A}$.

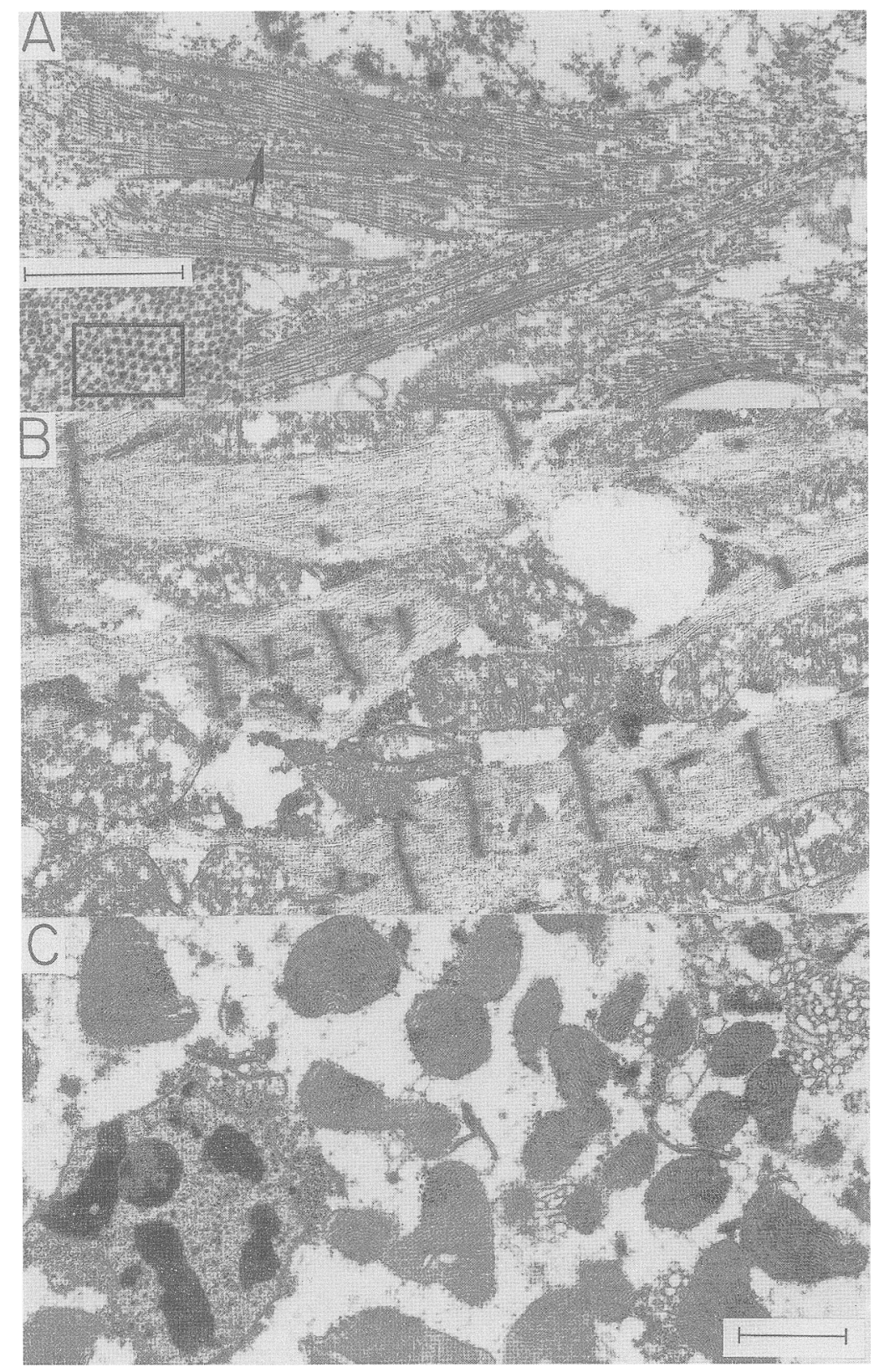


observed in single heterozygotes are due to filament imbalances, not deficits. We discuss these results in the light of models for myofibril assembly.

\section{Results}

We examined flight muscles of mutants having actin or myosin heavy chain null alleles in order to evaluate contributions of thick and thin filaments to the process of myofibril assembly (for a description of the strains used, see Materials and methods|. Representative electron micrographs are displayed in Figure 1. Myofibrils of Act $88 F^{K M 88}$ actin gene null allele homozygotes are illustrated in Figure 1A. No thin filaments or $\mathrm{Z}$ discs are present: Myofibrils consist of loosely organized skeins of thick filaments. $M$ lines, formed by proteins that crosslink thick filaments at their centers, and bare zones, central portions of thick filaments that lack myosin heads, appear normal. This demonstrates that thin filaments are not required for lateral registration of thick filaments or for their conjugation to $\mathrm{M}$ line proteins. As seen in cross sections (see Fig. 1A insert), thick filaments occasionally are found in well-ordered hexagonal arrays. Sections of the thick filaments that are so precisely packed always have electron-dense cores, which are known to occur only in central regions immediately adjacent to $M$ lines. This observation suggests that the $M$ line organizes or stabilizes the hexagonal packing pattern. Figure 1B illustrates myofibrils of $M h c 36 B^{\text {Ifm(2)2 }}$ myosin heavy chain null allele homozygotes. No thick filaments are present: Myofibrils consist of apparently continuous thin filament arrays anchored aperiodically within $\mathrm{Z}$ discs. Thus, in the absence of thick filaments, reasonably well-ordered thin filament/ $Z$ disc assemblies are able to form, but associations with thick filaments must be necessary for thin filament length determination and alignment, as well as precise $\mathrm{Z}$ disc spacing. Figure 1C illustrates $M h c 36 B^{I f m(2) 2}$; Act $88 F^{K M 88}$ double null homozygotes. In the absence of both thick and thin filaments, mitochondria and nuclei are the only recognizable organelles. Large sarcoplasmic voids are seen in place of myofibrillar arrays. In the absence of myofibrils, we see little evidence for an orderly system of $T$ tubules and sarcoplasmic reticulum, presumably because articulation of this membrane system depends on myofibril formation (Shafiq 1964).

Next, we examined flight muscles of actin and myosin heavy chain null mutation heterozygotes. To test whether the dominant flight muscle defects associated with actin and myosin heavy chain null alleles were due to imbalances in or deficits of actin and myosin accumulation, we produced flies that were heterozygotes for both $M h c 36 B^{I f m(2) 2}$ and $A c t 88 F^{K M 88}$ and compared their flight ability to that of both single null allele heterozygotes. If defects were due to an imbalance in protein or filament stoichiometry, the phenotype should improve in the double null heterozygote, because the ratio of functional actin and myosin genes $(1: 1)$ would be identical to that of wild-type $(2: 2)$. On the other hand, if muscle defects were due to protein or filament underproduction, they should be unimproved or aggravated in the double heterozygote. Figure 2 summarizes flight tests of four mutant genotypes and a wild-type strain. Figure 2A illustrates the testing apparatus, modeled after that used by Koana and Hotta (1978), a plexiglass cylinder with adhesive coating on its sides. When flies are released into the top portion of the tube, those capable of flight light within the upper levels, whereas those unable to fly drop to the lower levels. A histogram showing a typical profile obtained using wild-type flies is illustrated in Figure $2 \mathrm{~B}$. Over $90 \%$ of flies are recovered in the upper three levels $(1,2,3)$. As a negative control, we tested Act $88 F^{K M 88}$ homozygotes, which lack actin in their flight muscles. Over three quarters of these flies are recovered within the bottom level of the flight tester. Note that even without functional indirect flight muscles, nearly $25 \%$ of flies manage to reach the sides of the flight tester in levels 1-4, due largely to random trajectories of their falls. Figures $2 \mathrm{D}$ and E, illustrate flight performance of $M h c 36 B^{I f m(2) 2}$ and Act $88 F^{K M 88}$ heterozygotes, respectively. Myosin null allele heterozygotes fly very poorly, with $55 \%$ recovered in level 5 and $<5 \%$ recovered in level 1. Actin null allele heterozygotes are better fliers than myosin null allele heterozygotes, with
A)

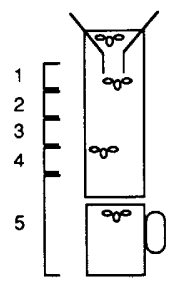

C) Act88F-
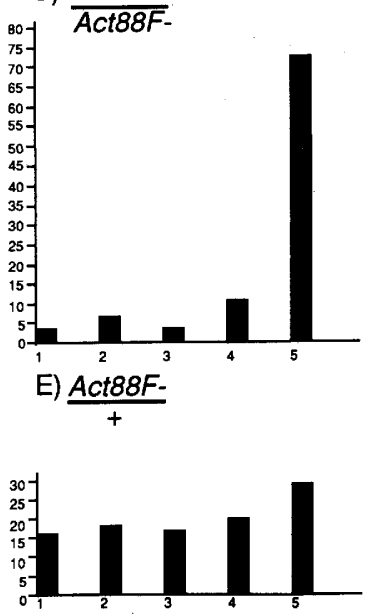

B) wild-type
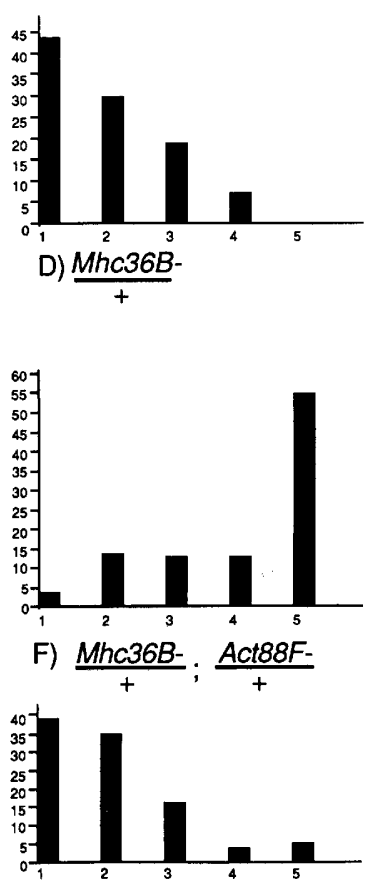

Figure 2. Flight tests of null mutation heterozygotes and controls. (A) Schematic diagram of the flight testing apparatus, modeled after that of Koana and Hotta (1978). Individuals introduced into the top of the cylinder fall to the bottom if flightless or light on the sides if flighted. Flies having normal flight muscles tend to light within the upper portions of the cylinder (levels one or two), whereas marginal or poor fliers tend to light in lower levels. $(B-F)$ Flight test results for indicated genotypes. The $\mathbf{x}$ coordinates signify the levels of the flight testing cylinder; the $y$ coordinates are the percentages of total flies collected in that level. At least 200 flies were tested to prepare each histogram. 
only $30 \%$ recovered in level 5 , but nevertheless perform poorly compared to wild type. Heterozygotes for both actin and myosin mutations are better fliers than either of the single null allele heterozygotes, as can be seen in Figure 2F. The performance of these flies approaches, but does not equal, that of wild type. Thus, the flightless phenotypes associated with single heterozygous null mutations cannot be caused by protein and filament deficits. Rather, the majority of defects must be caused by imbalances in accumulation of actin and myosin, or of thick and thin filaments.

We examined thin sections of actin and myosin heavy chain null allele heterozygote indirect flight muscles in order to further document how imbalances of the respective proteins affect myofibril formation. Figure 3 illustrates transverse sections of wild-type and mutant myofibrils. A section of wild-type flight muscles is shown in Figure 3A. Myofibrils contain thick and thin filaments that are aligned in a precise hexagonal array, with three thin filaments for every thick filament. This pattern has been described previously (Shafiq 1963b; Crossley 1978). Wild-type myofibrils measure 35-36 thick filaments at their widest point. Figure 3B shows Act $88 F^{K M 88} /$ + myofibrils. Within their central regions, thick and thin filaments are precisely ordered in hexagonal arrays, whereas thick filaments not integrated within the lattice are found around the periphery. The regions having precise lattice arrangement are one-half to two-thirds the wild-type myofibril diameter, typically measuring 18-24 thick filaments. Figure $3 \mathrm{C}$ illustrates a transverse section of flight muscles from $M h c 36 B^{\text {Ifm(2)2 }}$ heterozygotes. Myofibrils are grossly abnormal: They appear to be aggregates of small, well-organized arrays of thick and thin filaments. Surrounding each such array, we can discern supernumerary thin filaments. T-tubule/ sarcoplasmic reticulum dyads, typically located at the surface of the myofibril, are regularly found surrounding the small filament lattices within myofibrils of Mhc $36 B^{I f m(2) 2 / ~+~ h e t e r o z y g o t e s . ~ M o s t ~ m y o f i b r i l s ~ f r o m ~}$ this genotype are 25-30 thick filaments across, although some are much larger, like one pictured in the lower part of Figure 3C. Figure 3D shows myofibrils

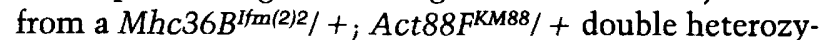
gote. Most myofibrils in this genotype are thinner than wild-type, typically $28-30$ thick filaments across, and somewhat less round. In $\sim 90 \%$ of these myofibrils, the entire filament lattices are packed perfectly. The remaining $10 \%$ either have breaks in filament packing similar to those seen in $M h c 36 B^{I f m(2) 2} /+$ heterozygotes, although far less severe, or consist of two small and well-arranged filament arrays separated by a region of electron-dense material. We have examined sections of flight muscles from several $M h c 36 B^{I f m(2) 2} /+$, Act $88 F^{K M 88 /}$ + double heterozygotes and find that myofibrils are considerably more normal than those of either single heterozygote in every case. These structural observations are consistent with previously described flight testing results.

In longitudinal sections of null allele heterozygote flight muscles, we see evidence for the same syndromes of defects apparent in transverse sections and discern additional abnormalities. Representative electron micrographs are displayed in Figure 4. Myofibrils of wild-type (Canton-S) indirect flight muscles are shown in Figure 4A. Note that thick filaments extend the length of the sarcomere and thus lack I-bands. Orderly registration and interdigitation of thick and thin filaments is demonstrated by regular spacing of $Z$ discs and by Moire effects caused by misalignment of the plane of the section with those of filament lattices. Myofibrils of Act $88 \mathrm{~F}^{\mathrm{KM}} \mathrm{B8} /+$ heterozygotes are organized as precisely repeated sarcomeres (Fig. 4B), although they are thin relative to wildtype myofibrils. The principal abnormality visible in longitudinal sections is the same as that seen in transverse sections: Disorganized thick filaments surround the myofibrillar lattice. In longitudinal sections of Mhc36 $B^{\text {Ifm(2)2/ }}+$ heterozygotes (Fig. $4 \mathrm{C}$ ), gaps or breaks between precisely interdigitated portions of myofibrils are apparent, giving them a torn appearance. In these sections, two facets of the mutant syndrome not visible in transverse sections can be seen: Small filament arrays frequently bridge two adjacent myofibrils, giving them a branched appearance, and very short sarcomeres are occasionally ligated to those having normal length. In comparison to myofibrils of either myosin heavy chain or actin null allele heterozygotes, those of Mhc36B Ifm(2)2/ + ; Act $88 F^{K M 88 /}+$ double heterozygotes appear relatively normal (Fig. 4D). The only departures from normalcy consistently seen are that double heterozygote myofibrils are not as straight as those of wildtype, and peripheral regions of $Z$ discs sometimes appear duplicated or bifurcated. These minor structural defects are most likely caused by reductions of actin and myosin heavy chain levels relative to other myofibrillar components, e.g. Z-disc proteins.

Finally, we measured sarcomere length in flight muscles of null allele heterozygotes in order to establish whether length is affected by stoichiometry of actin and myosin heavy chain. In wild-type muscle, thick filaments extend for the full length of the sarcomere, whereas thin filaments are approximately one-half this length. Filament lengths and, hence, sarcomere lengths, are invariant in wild-type flight muscles. We measured the average lengths of sarcomeres in wild-type myofibrils and in those of each mutant heterozygote by measuring Z-disc to Z-disc repeat distances in phase micrographs of macerated muscles. These data are summarized in Table 1. We find that $M h c 36 B^{1 f m(2) 2 /+}$ sarcomeres are somewhat shorter than those of wild

Table 1. Effects of actin/myosin ratio on sarcomere length

\begin{tabular}{|c|c|}
\hline Mutant & Length ( \pm S.E.M.) \\
\hline$r y^{506}$ (wild type) & $3.18 \pm 0.05 \mu \mathrm{m}$ \\
\hline$M h c-1+$ & $3.01 \pm 0.05$ \\
\hline Act $88 F-1+$ & $3.37 \pm 0.02$ \\
\hline Mhc- $1+; A c t 88 F-1+$ & $3.16 \pm 0.05$ \\
\hline
\end{tabular}

For a description of methodology employed, please see Experimental procedures. 


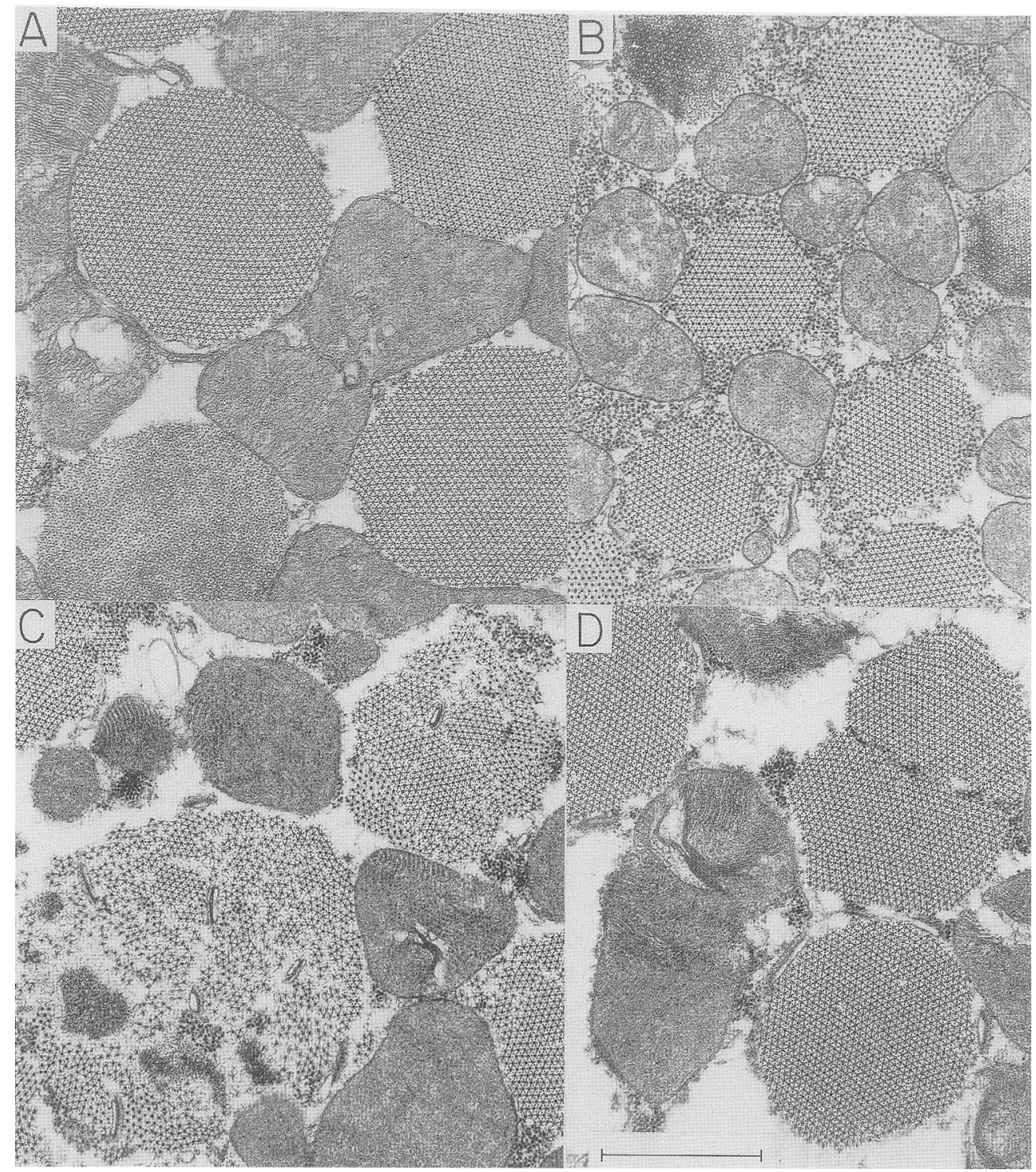

Figure 3. Transverse sections of flight muscles from actin and myosin heavy chain null allele heterozygotes. $(A)$ Cross sections of myofibrils from wild-type IFM. Myofibrils consist of cylindrical arrays, $\sim 1.5 \mu \mathrm{m}$ in diameter, of hexagonally packed thick and thin filaments. $(B)$ Myofibrils from $A c t 88 F^{K M 88} /+$ actin gene null allele heterozygotes. Myofibrils have well-packed lattices, but many unintegrated thick filaments are present around their periphery. Myofibril diameter is usually $<1 \mu \mathrm{m}$. (C) Myofibrils from

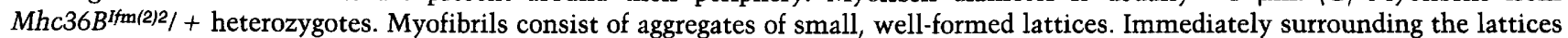
are unintegrated thin filaments and, occasionally, dyads of T-tubule/sarcoplasmic reticulum. Although myofibril diameter in this genotype is typically $1-1.2 \mu \mathrm{m}$, much larger myofibrils are apparent, as seen in the lower portion of $C$. $(D)$ Myofibrils of $M h c 36 B^{I f m(2) 2} /+$; Act $88 F^{K M 88} /+$ double heterozygotes. Myofibril lattices are well packed and average $1.2 \mu \mathrm{m}$ in diameter. Occasionally, double myofibrils are seen, apparently resulting from the fusion of two lattices, but myofibril morphology is otherwise remarkably normal. Bar, $1 \mu \mathrm{m}$.

type, whereas those of $A c t 88 F^{K M 88} /+$ are somewhat longer. Sarcomeres of $M h c 36 B^{I f m(2) 2 /+; A c t 88 F^{K M 88} /+}$ double heterozygotes are almost exactly the same length as wild type. Therefore, we find that protein or filament stoichiometry significantly influences sarcomere length determination. Reduction of myosin, relative to actin, shortens sarcomeres, whereas reduction of actin increases their length.

Occasionally we find considerably more dramatic al- terations of sarcomere length in flight muscles of $M h c 36 B^{I f m(2) 2} /+$ and $M h c 36 B^{\text {Ifm(2)2 } /+; A c t 88 F^{K M 88} /+}$ mutants. Figure 5, A and B, illustrate longitudinal sections of myofibrils from $M h c 36 B^{\operatorname{Ifm}(2) 2} /+$ flight muscles. In both micrographs, 'minisarcomeres' within the myofibrillar lattice can be discerned. Most frequently these occur within peripheral regions of myofibrils and are typically one-third the normal sarcomere length. Rarely (as in the lower right portion of Fig. 5A), a one-third- 


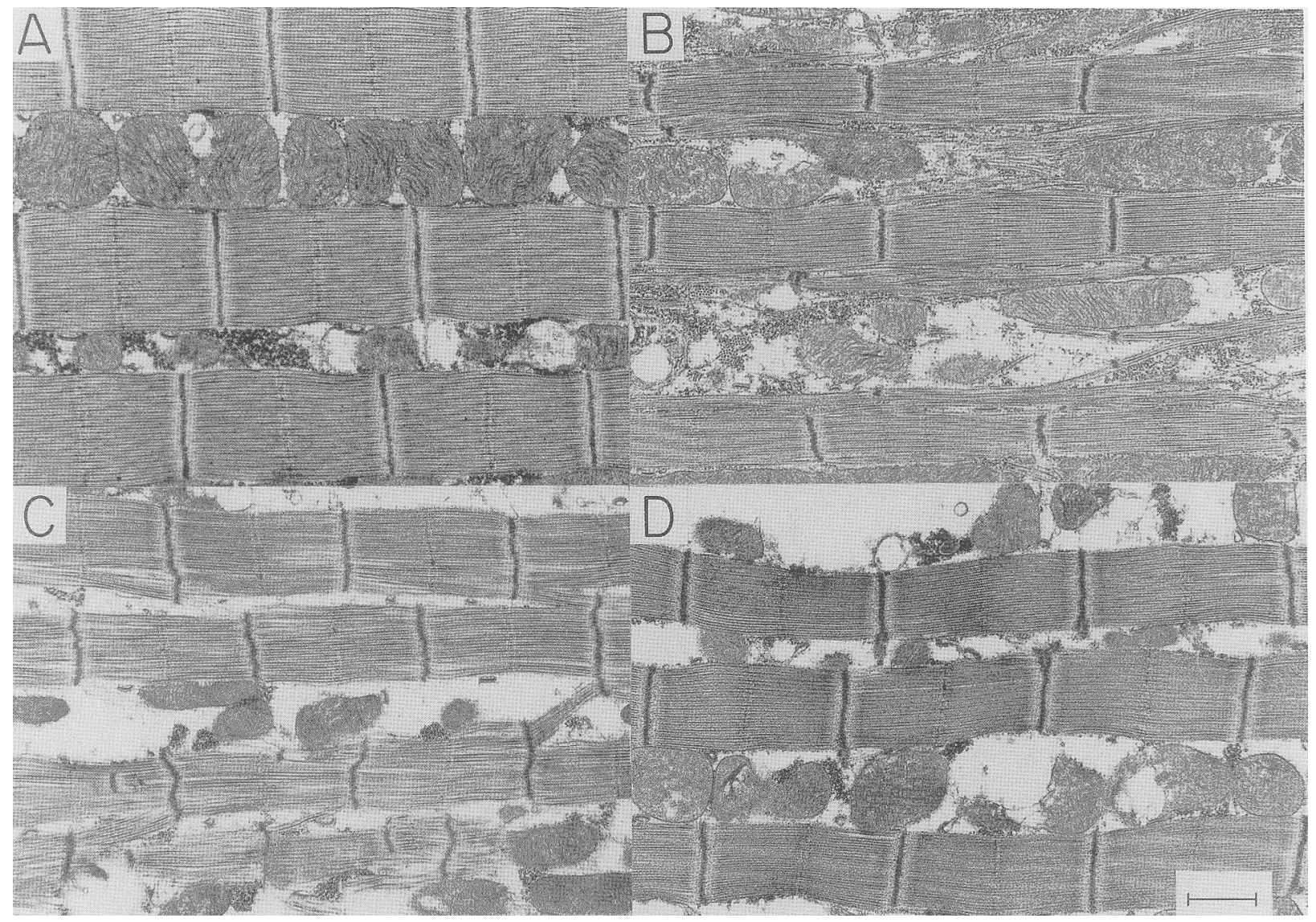

Figure 4. Longitudinal sections of flight muscles from myosin heavy chain and actin gene null allele heterozygotes. $(A)$ Myofibrils from wild-type IFM. Sarcomere length is typically $2.5 \mu \mathrm{m}$. $(B)$ Myofibrils from $A c t 88 F^{K M 88} /+$ heterozygotes. Sarcomeres are slightly longer than those of wild type. In longitudinal sections the unintegrated peripheral thick filaments are more readily apparent than in transverse sections. $(C)$ Myofibrils from $M h c 36 B^{l f m(2) 2} /+$ heterozygotes. As can be seen by comparison of $A$ and $C, M h c 36 B^{1 f m(2) 2 /+}$ heterozygotes are shorter than those of wild type. Because these myofibrils consist of aggregates of small lattices with interstitial discontinuities, they appear torn. Filament arrays frequently extend between adjacent myofibrils, forming branches. Finally, note the frequent occurrence of minisarcomeres-small filament arrays organized as sarcomeres ligated to mature myofibrils. $(D)$ Myofibrils from $M h c 36 B^{1 f m(2) 2 / ~+~} A c t 88 F^{K M 88 /}$ + double heterozygotes. Myofibrils are clearly more normal than those of either single heterozygote, although not as large or as well ordered as those of wild type. Bar, $1 \mu \mathrm{m}$.

length sarcomere extends the full width of the myofibril. In each case, the morphology of the minisarcomere appears normal; thick filaments taper at their ends, and $\mathrm{M}$ lines can be seen within central regions. Figure $5 \mathrm{C}$ illustrates myofibrils of $M h c 36 B^{I f m(2) 2 /+}$; Act $88 F^{K M 88} /+$ double heterozygotes. In 3 of 45 myofibrils examined, the sarcomere repeat length is significantly shorter than normal, as is the case for the lower of the illustrated pair of myofibrils. In these cases, the aberrant repeat length appears to be perpetuated along the entire course of the myofibril.

\section{Discussion}

Two significant conclusions can be drawn from our analyses of muscle defects engendered by actin and myosin heavy chain null alleles. The first is that assembly pathways for sarcomeric thick and thin filament arrays are remarkably independent. In the absence of one filament type, the other is able to form crude networks that resemble their sarcomeric arrangement. The second is that certain aspects of myofibril formation, most notably sarcomere length determination, circumferential growth of the filament lattice, and precise filament alignment, depend upon interactions of thick and thin filaments. This second conclusion is supported by the fact that filament alignment is poor in actin or myosin null allele homozygotes, as well as the observation that twofold alterations in ratios of functional actin and myosin heavy chain genes engender myofibrils wherein the former two parameters are defective. We emphasize that the majority of myofibrillar defects in single null allele heterozygotes cannot be due to protein or filament deficits, because $M h c 36 B^{I f m(2) 2} /+$; Act $88 F^{K M 88} /+$ double heterozygotes have considerably more normal myofibrils than does either single heterozygote. However, because myofibrils of double heterozygotes are not completely normal in either structural or functional senses, 


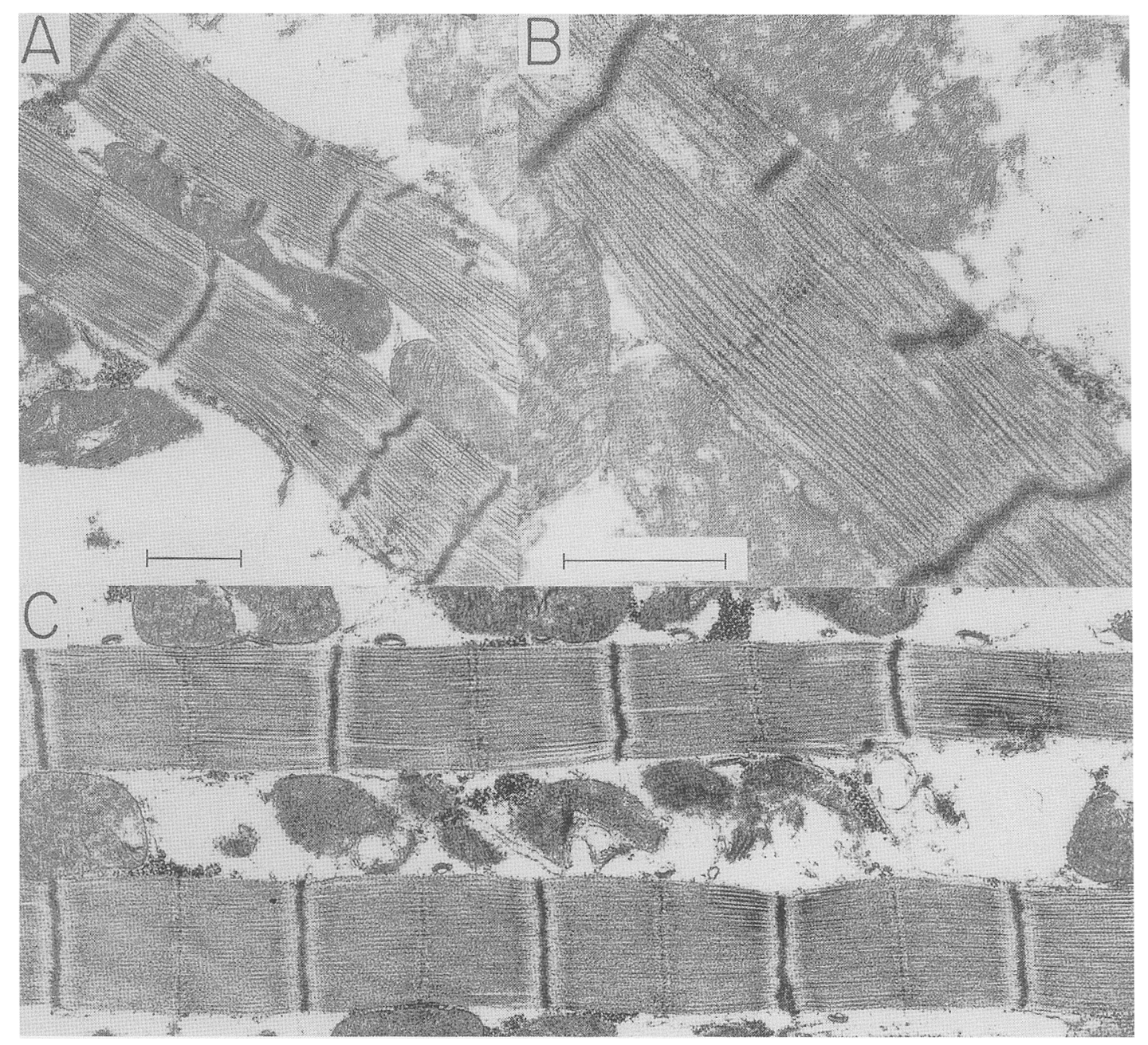

Figure 5. Defects in filament length determination observed in flight muscles of heterozygous null mutants. (A) Two adjacent myofibrils from a $M h c 36 B^{I f m(2) 2 /+h e t e r o z y g o t e, ~ b o t h ~ h a v i n g ~ m i n i s a r c o m e r e s . ~ I n ~ t h e ~ u p p e r ~ m y o f i b r i l, ~ t h e ~ s h o r t ~ s a r c o m e r e s ~ a r e ~ s e e n ~ i n ~}$ peripheral regions. The lower myofibril is a rare example wherein a sarcomere much shorter than normal extends across the myofibril

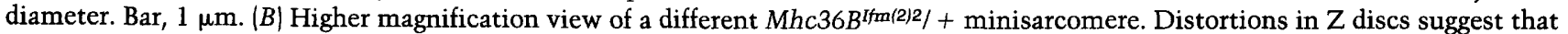

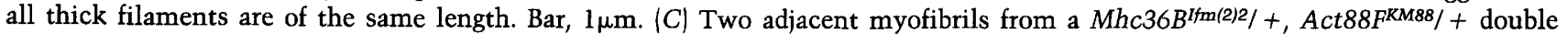
heterozygote. The lower myofibril of the pair has an atypically short sarcomere length that appears to be perpetuated throughout the entire length of the myofibril, whereas the sarcomere repeat length of the upper myofibril is precisely the same as in wild-type flight muscles. $(A)$ Bar, $1 \mu \mathrm{m}$ in $C$.

it remains possible that filament deficits contribute to the observed syndromes.

Previously reported immunocytochemical and electron microscopy studies of developing muscles have revealed several features of sarcomere formation. Vertebrate myofibrillogenesis begins with the assembly of microfilament networks immediately beneath the plasma membrane of embryonic myotubes (Peng et al. 1981; Dlugosz et al. 1984; Sanger et al. 1986). Some investigators have proposed that these networks give rise to nascent myofibrils having sarcomeres of the same length as those of mature myofibrils, either by direct conversion or serving as templates (Peng et al. 1981; Dlugosz et al. 1984), whereas others report that the nascent myofibrils are initially of small diameter and divided into short sarcomeres, which thereafter grow in girth and length (Sanger et al. 1986). The latter proposal is supported by several electron microscopy studies of developing invertebrate muscles (Aronson 1961; Shafiq 1963b; Auber 1969; Houlihan and Newton 1979/ each of which reported that sarcomeres in nascent myofibrils are two- to threefold shorter than mature sarcomeres.

Results reported here seem most compatible with models wherein initial microfilament arrays are nascent myofibrils, rather than templates for myofibril formation, and wherein sarcomeres lengthen as muscles develop. Specifically, the aperiodic distribution of $Z$ discs in $M h c 36 B^{I f m(2) 2}$ homozygotes is difficult to reconcile with the templating hypothesis, which would predict that $Z$ discs would be regularly spaced even in the absence of thick filaments. Likewise, the occurrence of minisarcomeres ligated to myofibril periphery in 
Mhc36B $B^{\text {Ifm(2)2 } / ~+~ h e t e r o z y g o t e s ~ i s ~ r e c o n c i l e d ~ m o s t ~ e a s i l y ~}$ with models wherein sarcomere length increases severalfold during myofibril maturation. One need only propose that lowering the thick/thin filament ratio predisposes myofibrils to grow circumferentially by extensive ligation of nascent myofibrils having short sarcomeres. Indeed, it is worth noting that the minisarcomeres observed in $M h c 36 B^{I f m(2) 2}$ myofibrils are the same size reported for nascent myofibrils of dipteran insects by several investigators (Shafiq 1963a,b; Auber 1969; Houlihan and Newton 1979).

Our results demonstrate that sarcomere formation requires both filament types and suggests that their proper stoichiometry is necessary as well. We have observed that networks of either thick or thin filaments assemble even when the complementary filament type is eliminated by null mutations, but note that these networks are not nearly as well ordered as in sarcomeres. We propose that thick and thin filament-anchoring proteins, most likely $\mathrm{M}$ line and $\mathrm{Z}$ disc proteins, respectively, organize these networks crudely, whereas myosin cross bridges serve to align them more rigorously. We further propose that proper thick and thin filament stoichiometry is essential for orderly circumferential growth of the filament lattice. Our evidence for this latter conjecture is that this aspect of myofibril growth is abnormal in both myosin heavy chain and actin null allele heterozygotes. Finally, interactions of actin filaments with thick filaments must be required to establish sarcomere length. This final hypothesis is based on the observation that thin filament networks of $M h c 36 B^{I f m(2) 2}$ homozygotes have aperiodically distributed $\mathrm{Z}$ discs.

To interpret muscle defects associated with actin and myosin heavy chain null allele heterozygotes more completely, it is desirable to understand the relationship of gene dosage to protein accumulation and genesis of the respective filament types. We have not quantified rates of actin and myosin heavy chain gene transcription and protein synthesis in flight muscles, but from ultrastructural observations it is clear that numbers of thick and thin filaments are reduced by $30-50 \%$ in the respective null allele heterozygotes. In hexagonally packed lattices of indirect flight muscles, the ratio of thin to thick filaments is always $3: 1$ (Crossley 1978). In both wild-type and $M h c 36 B^{\text {Ifm(2)2 } /+; A c t ~} 88 F^{K M 88 /}+$ double heterozygotes the ratio of thin to thick filaments must be exactly $3: 1$, because in both cases all discernible filaments are within lattices. However, the cross-sectional area of myofibrils in the double heterozygotes is only $60-70 \%$ that of wild type, and the total number of thin and thick filaments must be reduced accordingly. This apparent reduction of filament number precludes the notion that actin and myosin heavy chain levels are compensated in double heterozygotes, and this, in turn, leads to improved muscle phenotype. Myofibrils from single heterozygotes appear to have comparable reductions in numbers of respective filaments. In the myosin heavy chain null allele heterozygote, there are many thin filaments that are not integrated into myofibrillar lattices - the expected consequence of reducing thick filament number. In the actin null allele heterozygote, many unincorporated thick filaments are seen in peripheral regions of myofibrillar lattices-the expected consequence of reducing the number of actin filaments. In both $M h c 36 B^{I t m(2) 2} /+$ and $A c t 88 F^{K M 88} /+$ heterozygotes, myofibril cross-sectional areas are $30-50 \%$ less than that of wild type, and numbers of thick and thin filaments must be reduced in parallel.

Although both null allele heterozygotes have significant myofibrillar defects, adding extra copies of either $M h c 36 B$ or $A c t 88 F$ genes does not affect flight muscle function. Flies having one extra $M h c 36 B$ gene fly normally (Homyk and Emerson 1988), as do those having two extra Act $88 \mathrm{~F}$ gene copies introduced via P-elementmediated transformation (Hiromi et al. 1986; C. Beall and E. Fyrberg, unpubl.). The most plausible explanation is that actin and myosin are stable only if they can assemble with associated proteins. If amounts of these proteins were only sufficient for assembly of the normal number of thick and thin filaments, excess myosin or actin would be degraded. Support for this model is based on our observation of null mutations of Drosophila tropomyosin (Karlik and Fyrberg 1985) and troponin-T (C.C. Karlik and E.A. Fyrberg, in prep.) genes. Failure to produce either of these thin filament-associated proteins severely reduces levels of both actin and thin filaments. In summary, our analysis of flight muscle defects engendered by myosin heavy chain and actin null alleles has provided insights into some aspects of myofibril assembly and further demonstrates the utility of studying sarcomere formation using molecular genetic approaches. Extension of our work to several other contractile protein genes currently being characterized (for review, see Fyrberg 1989) and further developmental analyses of Drosophila muscle formation should ultimately allow formulation of a comprehensive explanation for sarcomere formation.

\section{Materials and methods}

Drosophila strains

The myosin heavy-chain mutants $M h c 36 B^{\operatorname{lfm}(2) 2}$ and $M h c 36 B^{b 85}$ were provided by Dr. S. Bernstein, and the actin mutant ry506 $A c t 88 F^{K M 88} e^{s}$ was provided by Dr. Y. Hotta. Other mutations and chromosomes are as described in Lindsley and Grell (1968) and Lindsley and Zimm (1982). A strain having the eye color marker $r y^{506}$ was used as wild type.

Singly heterozygous flies were generated by crossing the appropriate homozygotes to $\mathrm{ry}^{506}$. Double heterozygotes were generated by crossing $r y^{506} A c t 88 F^{K M 88} e^{s}$ homozygotes to either

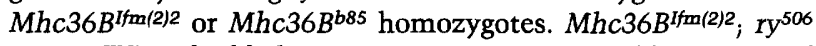
$A c t 88 F^{K M 88} e^{s}$ double homozygotes were generated by a series of crosses. $\mathrm{ry}^{506} \mathrm{Act} 88 \mathrm{~F}^{\mathrm{KM} 88} e^{s}$ homozygotes were crossed to $\mathrm{CyO}$; $r y / T M 6 b$ /the TM $6 b$ chromosome is marked with the dominant mutation $T b \mid$, and non-ry, $T b, C y$ female progeny were selected. $M h c 36 B^{I f m(2) 2}$ flies were crossed to $S c o$; ry/TM3 (the TM3 chromosome is marked with the dominant mutation $S b \mid$, and $S c o$; $S b$ male progeny were selected. The two groups of progeny were intermated and $C y, S b$, non- $S c O$, non- $T b$ flies were isolated. Their genotype is Mhc36 $B^{1 f m(2) 2} / C y O$; ry ${ }^{506}$ Act $88 F^{K M 88} e^{s / T M 3}$. These were kept as a stock, and double homozygotes (ry, non$C y$, non- $S b \mid$ progeny were selected. 
It is extremely unlikely that muscle defects associated with either of the null alleles are due to production of abnormal myosin heavy chain or actin. Genetic experiments have shown that flightless phenotypes associated with $M h c 36 B^{l f m(2) 2}$ and Act $88 F^{K M 88}$ heterozygotes can be rescued by single wild-type gene copies (Homyk and Emerson 1988; Hiromi et al. 1986). This observation demonstrates that the muscle defects cannot be due to antimorphic effects of mutant proteins. Furthermore, RNA blot-hybridization experiments have failed to reveal stable myosin heavy chain or actin transcripts in flight muscles

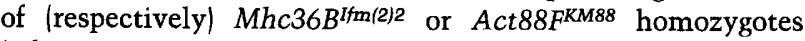
(Okamoto et al. 1986; Chun and Falkenthal 1988). The absence of respective transcripts confirms that both alleles are true null alleles. Finally, we note that in the myosin heavy chain null allele experiments, we used two different alleles, $M h c 36 B^{\text {Ifm(2)2 }}$ (Mogami and Hotta 1981) and $M h c 36 B^{b 85}$ (Bernstein, pers. comm.). We obtained identical results in both cases, further demonstrating that the phenotypes are due to absence of myosin, rather than to effects of mutant myosins.

\section{Flight tests}

Flies, 0-3 days old, were transferred to a fresh vial and aged for 1 day. Our flight testing apparatus was like that of Koana and Hotta (1978) and modified as described by Green et al. (1986). A plexiglass cylinder having a $6-\mathrm{cm}$ inner diameter was lined with an acetate sheet coated with Tangle-Trap insect-trapping adhesive (Carolina Biological Supply). Flies were shaken lightly through a standard powder funnel into the column. A beaker was used to collect flies that fell to the bottom. The acetate sheets were removed and placed over a grid to divide them into areas $7 \mathrm{~cm}$ wide, and the number of flies in each area was counted, as was the number collected in the bottom.

\section{Electron microscopy of IFM}

Our fixation and embedding protocols are based upon those of M. Reedy (Reedy and Reedy 1985, and pers. comm.). Whole thoraces were fixed in $3 \%$ glutaraldehyde, $0.2 \%$ tannic acid in buffered ringers [1 $10 \mathrm{mM} \mathrm{NaCl}, 2 \mathrm{mM} \mathrm{KCl}, 3 \mathrm{mM} \mathrm{MgCl}_{2}, 20 \mathrm{mM}$ K-MOPS [pH 6.8)] at $4^{\circ} \mathrm{C}$ overnight. Muscle fibers were dissected from thoraces in buffered Ringer's, rinsed once more in the same solution, and twice in $0.2 \mathrm{M} \mathrm{NaPO}_{4}(\mathrm{pH} 7.0)$. The fibers were postfixed in $1 \% \mathrm{OsO}_{4}, 0.2 \mathrm{M} \mathrm{PO}_{4}|\mathrm{pH} 7.0|$ for $1 \mathrm{hr}$ on ice, rinsed in $\mathrm{H}_{2} \mathrm{O}$ three times, stained in $2 \%$ uranyl acetate to 1 $\mathrm{hr}$ at room temperature, rinsed three times in $\mathrm{H}_{2} \mathrm{O}$, and dehydrated through an ethanol series. The fibers were infiltrated with Epon 812 /dodecenylsuccininc anhydride/araldite $(1: 7: 2$ by volumel, and the resin polymerized. Silver/gold sections were cut, stained with uranyl acetate and lead citrate, and observed in the electron microscope. Flight muscles of six different flies of each genotype were examined, and qualitatively similar morphology was found within each strain.

\section{Sarcomere length measurements}

IFM were dissected from adult flies of the appropriate genotypes in $128 \mathrm{~mm} \mathrm{NaCl}, 4 \mathrm{~mm} \mathrm{KCl}, 2 \mathrm{~mm} \mathrm{CaCl}, 10 \mathrm{~mm}$ HEPES $(\mathrm{pH}$ 6.9). Fibers were macerated with tungsten needles and photographed using a $63 \times$ phase objective. Sarcomere length was determined by direct measurements of projected images of myofibrils.

\section{Acknowledgments}

We thank Professors Yoshiki Hotta and Sanford Bernstein for providing mutants and Mary Reedy for suggesting improve- ments in our electron microscopy protocols and for informative discussions. We thank Drs. S. Falkenthal and S. Bernstein for communicating results prior to publication. We are especially grateful to Carnegie Institute of Washington for allowing us to use their electron microscopy facilities. This work was supported by grants from the National Institutes of Health and Muscular Dystrophy Association to E.A.F. C.J.B. was supported by a Postdoctoral Fellowship from the Muscular Dystrophy Association of America.

\section{References}

Aronson, J. 1961. Sarcomere size in developing muscles of a Tarsonemid mite. J. Biophys. Biochem. Cytol. 11: 147-156. Auber, J. 1969. La myofibrillonese du muscle strie. 1. Insectes. J. Micros. 8: 197-232.

Ball, E., C.C. Karlik, C.J. Beall, D.L. Saville, B. Bullard, and E.A. Fyrberg. 1987. Arthrin, a myofibrillar protein in insect flight muscle, is an actin-ubiquitin conjugate. Cell 51: 221-228.

Bernstein, S.I., K. Mogami, J.J. Donady, and C.P. Emerson. 1983. Drosophila myosin heavy chain encoded by a single gene in a cluster of muscle mutations. Nature 302: 393-397.

Bernstein, S.I., C.J. Hansen, K.D. Becker, D.R. Wassenberg, E.S. Roche, J.J. Donady, and C.P. Emerson. 1986. Alternative RNA splicing generates transcripts encoding a thorax-specific isoform of Drosophila melanogaster myosin heavy chain. Mol. Cell. Biol. 6: 2511-2519.

Crossley, A.C. 1978. The morphology and development of the Drosophila muscular system. In The genetics and biology of Drosophila, vol. 2B., (ed. M. Ashburner and T.R.F. Wright), pp. 499-560. Academic Press, New York.

Chun, M. and S. Falkenthal. 1988. Ifm(2)2 is a myosin heavy chain allele that disrupts myofibrillar assembly only in the indirect flight muscle of Drosophila melanogaster. I. Cell Biol. 107: 2613-2621.

Davis, J.S. 1988. Assembly processes in vertebrate skeletal thick filament formation. Annu. Rev. Biophys. Chem. 17: 217-239.

Dlugosz, A.A., P.B. Antin, V.T. Nachmias, and H. Holtzer 1984. The relationship between stress fiber-like structures and nascent myofibrils in cultured cardiac myocytes. /. Cell Biol. 99: 2268-2278.

Fischman, D.F. 1967. An electron microscopy study of myofibril formation in embryonic chick skeletal muscle. $/$. Cell Biol. 32: 557-575.

-1986. Myofibrillogenesis and the morphogenesis of skeletal muscle. In Myology (ed. A.G. Engel and B.Q. Bankerl, pp. 5-37. McGraw-Hill Book Company, New York.

Fyrberg, E. 1989. Study of contractile and cytoskeletal proteins using Drosophila genetics. Cell Motil. Cyto. (in press).

Green, C.C, J.C. Sparrow, and E. Ball. 1986. Flight testing columns. Dros. Inf. Serv. 63: 141.

Hiromi, Y., H. Okamoto, W.J. Gehring, and Y. Hotta. 1986. Germ line transformation with Drosophila mutant actin genes induces constitutive expression of heat shock genes. Cell 44: 293-301.

Homyk, T. and C.P. Emerson. 1988. Functional interactions between unlinked muscle genes within haploinsufficient regions of the Drosophila genome. Genetics 119: 105-121.

Houlihan, D.F. and J.R.L. Newton. 1979. Sarcomere formation and longitudinal growth in the developing flight muscle of Calliphora. J. Insect Physiol. 25: 879-893.

Karlik, C.C. and E.A. Fyrberg. 1985. An insertion within a variably spliced Drosphila tropomyosin gene blocks accumulation of only one encoded isoform. Cell 41: 57-66. 
Beall et al.

Koana, T. and Y. Hotta. 1978. Isolation and characterization of flightless mutants of Drosophila melanogaster. J. Embryol. Exp. Morph. 45: 123-143.

Lindsley, D.L. and E.H. Grell. 1968. Genetic variations of Drosophila melanogaster. Carnegie Inst. Washington Publ. No. 627.

Lindsley, D.L. and G. Zimm. 1982. Computerized stock. List 3. Dros. Inf. Serv. 57.

Mogami, K. and Y. Hotta. 1981. Isolation of Drosophila flightless mutants which affect myofibrillar proteins of indirect flight muscles. Mol. Gen. Genet. 183: 409-417.

O'Donnell, P.T. and S.I. Bernstein. 1988. Molecular and ultrastructural defects in a Drosophila myosin heavy chain mutant: Differential effects on muscle function produced by similar thick filament abnormalities. J. Cell Biol. 107: 2601-2612.

Okamoto, H., Y. Hiromi, E. Ishikawa, T. Yamada, K. Isoda, H. Maekawa, and Y. Hotta. 1986. Molecular characterization of mutant actin genes which induce heat-shock proteins in Drosophila flight muscles. EMBO I. 5: 589-596.

Peng, H.B., J.J. Wolosewick, and P-C. Cheng. 1981. The development of myofibrils in cultured muscle cells: A whole mount and thin-section electron microscopic study. Dev. Biol. 88: 121-136.

Pollard, T.D. and J. Cooper. 1986. Actin and actin binding proteins: A critical evaluation of mechanisms and functions. Annu. Rev. Biochem. 55: 987-1035.

Reedy, M.K., and M.C. Reedy. 1985. Rigor crossbridge structure in tilted single filament layers and flared-X formations from insect flight muscle. J. Mol. Biol. 185: 145-176.

Sanger, J.M., B. Mittal, M.B. Pochapin, and J.W. Sanger. 1986. Myofibrillogenesis in living cells microinjected with fluorescently labeled alpha-actinin. I. Cell Biol. 102: 20532066.

Shafiq, S.A. 1963a. Electron microscopic studies on the indirect flight muscles of Drosophila melanogaster. I. Structure of the myofibrils. J. Cell Biol. 17: 351-362.

1963b. Electron microscopic studies on the indirect flight muscles of Drosophila melanogaster. II. Differentiation of myofibrils. J. Cell Biol. 17: 363-373.

1964. An electron microscopical study of the innervation and sarcoplasmic reticulum of the fibrillar flight muscles of Drosophila melanogaster. I. Microsc. Sci. 105: 1-6.

Taylor, K.A., M.C. Reedy, L. Cordova, and M.K. Reedy. 1984. Three-dimensional reconstruction of rigor insect flight muscle from tilted thin sections. Nature 310: 285-291.

Waterston, R.H. and G.R. Francis. 1985. Genetic analysis of muscle development in Caenorhabditis elegans. Trends Neurosci. 8: 270-276. 


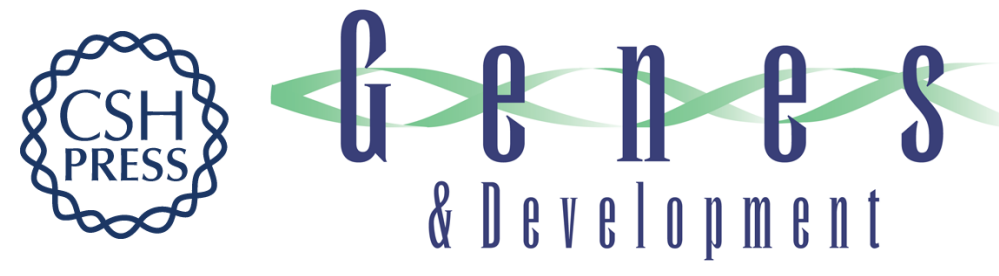

\section{Genetic dissection of Drosophila myofibril formation: effects of actin and myosin heavy chain null alleles.}

C J Beall, M A Sepanski and E A Fyrberg

Genes Dev. 1989, 3:

Access the most recent version at doi:10.1101/gad.3.2.131

References This article cites 27 articles, 9 of which can be accessed free at: http://genesdev.cshlp.org/content/3/2/131.full.html\#ref-list-1

License

Email Alerting

Service

Receive free email alerts when new articles cite this article - sign up in the box at the top right corner of the article or click here.

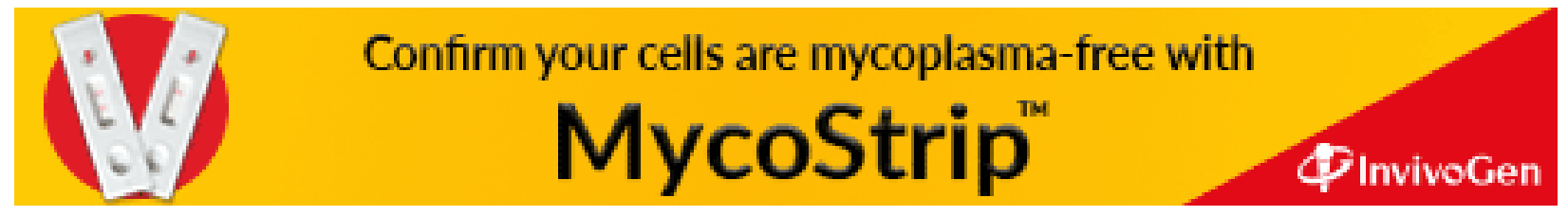

